\title{
Assessing Mitral Regurgitation in Patients with Dilated Cardiomyopathy Using 3.0T Cardiac Magnetic Resonance Imaging: Correlation with Morphological and Functional Abnormalities
}

\author{
Ling-yi Wen, ${ }^{1}$ Zhi-gang Yang, ${ }^{1,2,}{ }^{*}$ Ying-kun Guo, ${ }^{3}$ Jia-yu Sun, ${ }^{1}$ and Hua-yan $\mathrm{Xu}^{1,2}$ \\ ${ }^{1}$ Department of Radiology, West China Hospital, Sichuan University, Chengdu, China \\ ${ }^{2}$ National Key Laboratory of Biotherapy, West China Hospital, Sichuan University, Chengdu, China \\ ${ }^{3}$ Department of Medical Imaging, West China Second University Hospital, Sichuan University, Chengdu, China \\ "Corresponding author: Zhi-gang Yang, Department of Radiology, West China Hospital, Sichuan University, Chengdu, China. Tel/Fax: +86-28 85423817, E-mail: \\ yangzg666@163.com
}

Received 2016 June 16; Revised 2016 August 17; Accepted 2016 September 04.

\begin{abstract}
Background: Mitral regurgitation frequently accompanies dilated cardiomyopathy (DCM), which is associated with poor prognosis.

Objectives: To evaluate morphological and functional abnormalities in DCM patients and correlate these features with the severity of coexistent mitral regurgitation using 3.0 T magnetic resonance imaging (MRI).

Patients and Methods: Forty-one patients with DCM and 26 healthy control subjects underwent MRI and echocardiography examinations. The maximum and minimum mitral annulus areas (MAA), diameters of left ventricle and atrium, end-diastolic and systolic volumes, and ejection fraction were assessed with MRI and were indexed to body surface area. Mitral regurgitation severity grading was estimated by echocardiography.

Results: Of the 41 patients with DCM, echocardiography examination revealed 11 patients (27\%) without mitral regurgitation and 12 (29\%) with mild, 11 (27\%) with moderate, and seven (17\%) with severe mitral regurgitation. All of the morphological and functional parameters of left ventricle, atrium and mitral valve in DCM patients were greater than those in healthy subjects (all $\mathrm{P}<0.05$ ), except for ejection fraction. The indexed end-systolic diameters of left ventricle (LVESD) and atrium (LAESD) and indexed minimum MAA might have the capability to distinguish no/mild mitral regurgitation patients from moderate/severe patients (areas under the receiver operating characteristic curve $[\mathrm{AUC}]=0.876,0.816$, and 0.773 , respectively; all $\mathrm{P}<0.05)$. Using a combination of indexed LAESD, LVESD and minimum MAA, the specificity increased to $91.3 \%$.

Conclusion: Mitral regurgitation in DCM patients is associated with morphological and functional abnormalities of left heart and mitral valve annulus. The indexed LVESD, LAESD, and minimum MAA values can assist in predicting the severity of mitral regurgitation with a high sensitivity and specificity.
\end{abstract}

Keywords: Magnetic Resonance Imaging, Cardiomyopathy, Dilated, Mitral Valve Insufficiency, Left Ventricular Function

\section{Background}

Mitral regurgitation frequently accompanies dilated cardiomyopathy (DCM), which is associated with a poor prognosis (1). The increasing severity of mitral regurgitation, especially in moderate/severe patients remains an independent predictor of heart failure and sudden cardiac death $(2,3)$. Because DCM has multiple etiologies and morphological features of the disease, the detailed mechanism for coexistence of mitral regurgitation in DCM is complex and controversial. Several studies have shown that mitral regurgitation in DCM patients is caused by left ventricle (LV) and left atrium (LA) dysfunction and mitral valve complex abnormalities (4-6). However, whether the severity of mitral regurgitation in DCM patients correlates with the morphological and functional abnormalities of the left heart is uncertain.

With its high spatial and temporal resolution, magnetic resonance imaging (MRI) is regarded as the gold standard for the assessment of cardiac function and structure. Compared to 1.5 T MRI, 3.0 T MRI increases the signal-tonoise ratio (SNR) for the myocardium and blood, allowing accurate assessment of ventricular and atrial dysfunction, visualization and quantification of the morphology of the mitral valve $(7,8)$. These advantages can overcome the limitations of conventional echocardiography, such as the low spatial resolution and significant observer variability, which can affect the measurements' reproducibility and credibility (9). The clinical LV function parameter data of DCM patients have been well documented by echocardiography (10); whereas, to our best knowledge, morphological and functional abnormalities on 3.0T MRI that may assist 
in prediction of mitral regurgitation severity are lacking.

\section{Objectives}

The aim of this study was to evaluate whether 3.0T MRI could estimate the correlation between mitral regurgitation and morphological, functional abnormalities of the left heart in DCM patients and whether this could assist in the prediction of the severity of mitral regurgitation.

\section{Patients and Methods}

\subsection{Patient Selection}

The study was approved by the institutional review board of our medical school. Written informed consent was obtained from all patients and controls.

From January 2014 to February 2016, we examined 47 consecutive DCM patients who underwent cardiac MRI at our medical center. The diagnosis of DCM was based on increased LV dimensions (left ventricular end-diastolic diameter $\geq 3.2 \mathrm{~cm} / \mathrm{m}^{2}$ ) (11), the presence of a globally decreased contraction pattern (the contraction pattern is assessed using a grading system: hyperkinesia, increased wall motion; normokinesia, normal wall motion toward the center of the ventricle; hypokinesia, decreased wall motion; akinesia, absent wall motion. Hypokinesia and akinesia are decreased contraction patterns)(12) measured by echocardiography, and evidence of normal coronary arteries on coronary angiography or coronary computed tomography angiography. The exclusion criteria consisted of the presence of organic heart valvular disease or other combined cardiovascular diseases $(n=13)$, an incomplete MRI examination $(\mathrm{n}=1)$, and non-interpretable MRI image quality $(n=2)$. Thus, 41 patients (mean age, $43 \pm 18$ years; range, 15 to 79 years) were enrolled in the study and underwent two-dimensional (2D) transthoracic echocardiography (TTE) and MRI. These patients included 29 men (mean age, $41 \pm 16$ years; range, 16 to 74 years) and 12 women (mean age, $47 \pm 20$ years; range, 15 to 79 years). No significant difference was found in age between the men and women $(\mathrm{P}=0.40)$.

\subsection{MRI}

All patients were examined with a 3.0-T whole-body scanner (Trio Tim; Siemens medical solutions, Erlangen, Germany) with a maximum gradient strength of $50 \mathrm{mT} / \mathrm{m}$ and a maximum flew rate of $200 \mathrm{mT} / \mathrm{m} / \mathrm{ms}$. Electrocardiographic gating and breath holding in expiration were used when feasible. Long-axis two-, three-, and four-chamber images were acquired with a segmented echocardiographygated Turbo FLASH cine sequence (slice thickness: $8 \mathrm{~mm}$, repetition time: $37.66 \mathrm{~ms}$, echo time: $1.2 \mathrm{~ms}$, flip angle: $50^{\circ}$ ). Data acquisition was performed during end-inspiratory breath holding. For calculation of functional parameters, 8 - 12 serial short-axis slices from the base to the apex were acquired with an echocardiography-gated Turbo FLASH cine sequence (slice thickness: $10 \mathrm{~mm}$, repetition time: 37.66 $\mathrm{ms}$, echo time: $1.2 \mathrm{~ms}$, flip angle: $50^{\circ}$, spacing between slices: $0 \mathrm{~mm}$ ). Furthermore, the planimetry of the mitral annulus area was measured on the basal slice of the shortaxis, which was demonstrated to be the mitral valve annulus.

\subsection{Echocardiography}

2D TTE and MRI were performed within 7 days with a multi-planar 1.5 - $3 \mathrm{MHz}$ probe (IE33; Philips medical systems, Andover, Mass). With the patient in left lateral decubitus position, images were acquired in the parasternal, subcostal, and apical two-, three-, and four-chamber views for assessment of the degree of mitral regurgitation. The mitral regurgitation grading was classified with a threepoint scale based on the ratio of absolute area of regurgitation jet to the size of the left atrium (mild, less than $20 \%$; moderate, $20 \%$ - 40\%; severe, greater than $40 \%$ ) (13). Because the prognosis and clinical treatments are quite different with different mitral regurgitation grades, especially in moderate/severe mitral regurgitation patients (3, 14), we divided DCM patients into two subgroups: no/mild mitral regurgitation and moderate/severe mitral regurgitation.

\subsection{Image Analysis}

For MRI images, LV volumes and ejection fraction (EF) were measured with commercially available software (Argus; Siemens Medical Solutions) using serial short-axis slices. The endo-and epicardial borders were traced during the end-diastole and end-systole to obtain the volumetric parameters with the section summation method. For LA end-diastolic and end systolic volume (LAEDV, LAESV), both end-diastolic and end-systolic contours were traced in twochamber and four-chamber views and calculated using biplane area-length method (15).

The LV and LA dimensions were determined on fourchamber views (Figure 1). The LV dimensions were measured as the maximal distance between the interventricular septum and lateral ventricular endocardium, which was perpendicular to the long axis in end-systole and enddiastole. The LA dimensions were defined as the maximal distance perpendicular to atrial length in end-systole and end-diastole, while the atrial length was measured as the distance between the midwall of the left atrium and the midpoint of the mitral annulus. The maximum and minimum mitral annulus areas $\left(\mathrm{MAA}_{\max }\right.$ and $\left.\mathrm{MAA}_{\min }\right)$ were 
planimetered at the mitral valve annulus slice (Figure 2). The volumes and dimensions of the LV, LA, MAA max and $\mathrm{MAA}_{\min }$ were indexed to body surface area (BSA).

\subsection{Variability Analysis of MRI Measurements}

All of the MRI images were analyzed initially by one experienced radiologist who was blinded to the clinical data. To control intraobserver variability, the image analysis was repeated by the same investigator 1 month later. To control interobserver variability, a second experienced investigator independently reanalyzed the measurements without prior knowledge of the clinical data.

\subsection{Statistical Analysis}

Continuous and categorical variables were expressed as means \pm SD and numbers (percentages), respectively. For all continuous categories, skewness and kurtosis were used to test for normality, and Levene's test was used to test for homogeneity of variance. Comparisons of two normally distributed variables were performed with Student's t test; otherwise, Kruskal Wallis tests were used. The intraclass correlation coefficient (ICC) was used to calculate the interobserver and intraobserver variability. DCM patients were divided into four groups based on the presence and grading of mitral regurgitation. Correlation between mitral regurgitation grading and MRI parameters was assessed with Spearman's rank correlation analysis. Receiver operating characteristic analysis was used to predict the severity of mitral regurgitation. All statistical analyses were performed using SPSS software (version 16.0 for windows; SPSS Inc., Chicago, Illinois, USA). A 2-tailed Pvalue $<0.05$ was considered statistically significant.

\section{Results}

\subsection{Patient Characteristics}

A total of 41 DCM patients were eligible for the study (Table 1). Echocardiographic examinations revealed 11 patients $(27 \%)$ without mitral regurgitation and 12 patients (29\%) with mild, 11 (27\%) with moderate, and 7 (17\%) with severe mitral regurgitation. According to the New York heart association (NYHA) classification, 5 (12\%), 8 (20\%), 16 (39\%) and 12 (29\%) patients had a class of I, II, III, and IV, respectively. No differences in mitral, LA and LV parameters were evident among DCM patients with respect to the NYHA class.
4.2. Morphological and Functional Abnormalities of the Left Heart and Mitral Valve

Data for the parameters of the mitral valve, LA and LV are summarized in Table 2. The EF values of DCM patients were significantly lower than those of healthy subjects $(23.6 \pm 11.7 \%$ vs. $61.3 \pm 6.6 \%, \mathrm{P}<0.001)$. The indexed enddiastolic volume (EDV) and end-systolic volume (ESV), the indexed end-diastolic dimension (EDD) and end-systolic dimension (ESD) of the LV and LA were significantly higher in patients than in control subjects (all $\mathrm{P}<0.005$ ). In DCM patients, both the indexed $\mathrm{MAA}_{\min }\left(9.9 \pm 4.0 \mathrm{~cm}^{2} / \mathrm{m}^{2}\right.$ vs. $\left.5.9 \pm 1.2 \mathrm{~cm}^{2} / \mathrm{m}^{2}, \mathrm{P}=0.001\right)$ and indexed $\mathrm{MAA}_{\max }(12.9 \pm 3.8$ $\mathrm{cm}^{2} / \mathrm{m}^{2}$ vs. $9.5 \pm 1.1 \mathrm{~cm}^{2} / \mathrm{m}^{2}, \mathrm{P}=0.002$ ) were significantly larger than in healthy subjects.

\subsection{Correlation Between Mitral Regurgitation Grading and Morphological, Functional Abnormalities}

Within the DCM group, the Spearman correlation test indicated a good correlation between grading of mitral regurgitation and the function. Morphological parameters are listed in Table 3. They show a good correlation $(r=0.43$ to 0.61 ; all $\mathrm{P}<0.05$ ) but not for EF values $(\mathrm{r}=-0.38)$. The indexed LVESD, LAESD and MAA min $_{\text {mend }}$ te increase with increasing grades of mitral regurgitation $(r=0.58,0.61$, and 0.56 , respectively, all $\mathrm{P}<0.001$ ). Moreover, a significant correlation was found between indexed LVESD and LAEDD in patients with $\mathrm{DCM}(\mathrm{r}=0.92 ; \mathrm{P}<0.001)$. A moderate but significant correlation was evident between indexed $M A A_{\text {min }}$ and LAEDD $(\mathrm{r}=0.47, \mathrm{P}=0.004)$, and indexed $\mathrm{MAA}_{\min }$ and $\operatorname{LVESD}(\mathrm{r}=0.45, \mathrm{P}=0.005)$.

According to the above-mentioned criteria, 23 patients (56\%) had no/mild mitral regurgitation, and 18 patients (44\%) had moderate/severe mitral regurgitation. Patients with moderate/severe mitral regurgitation showed lower BSA and BMI values than that of no/mild mitral regurgitation patients $(\mathrm{P}<0.05)$. The MRI parameters of two subgroups are listed and compared in Table 3. All morphological and functional parameters of LV, LA, and mitral valve in DCM patients with moderate/severe mitral regurgitation were greater than that of no/mild mitral regurgitation patients (all $\mathrm{P}<0.05)$, except for EF values $(19.3 \pm 7.5 \%$ vs. $26.6 \pm 13.5 \%, \mathrm{P}<0.05)$. As shown by diagnostic statistics in Table 4, the indexed LVESD, LAESD and MAA $A_{\text {min }}$ might be used to distinguish no/mild mitral regurgitation patients from moderate/severe mitral regurgitation patients with high sensitivity and specificity (areas under receiver operating characteristic curve $[$ AUC $]=0.876,0.816$, and 0.773 , respectively, all $\mathrm{P}<0.05$ ) (Figure 3 ). When a single parameter was used, a relatively higher AUC (0.816), sensitivity (88.9\%) and specificity (82.6\%) could be achieved with a cutoff of 3.2 $\mathrm{cm} / \mathrm{m}^{2}$ for indexed LAESD. The highest negative predictive 

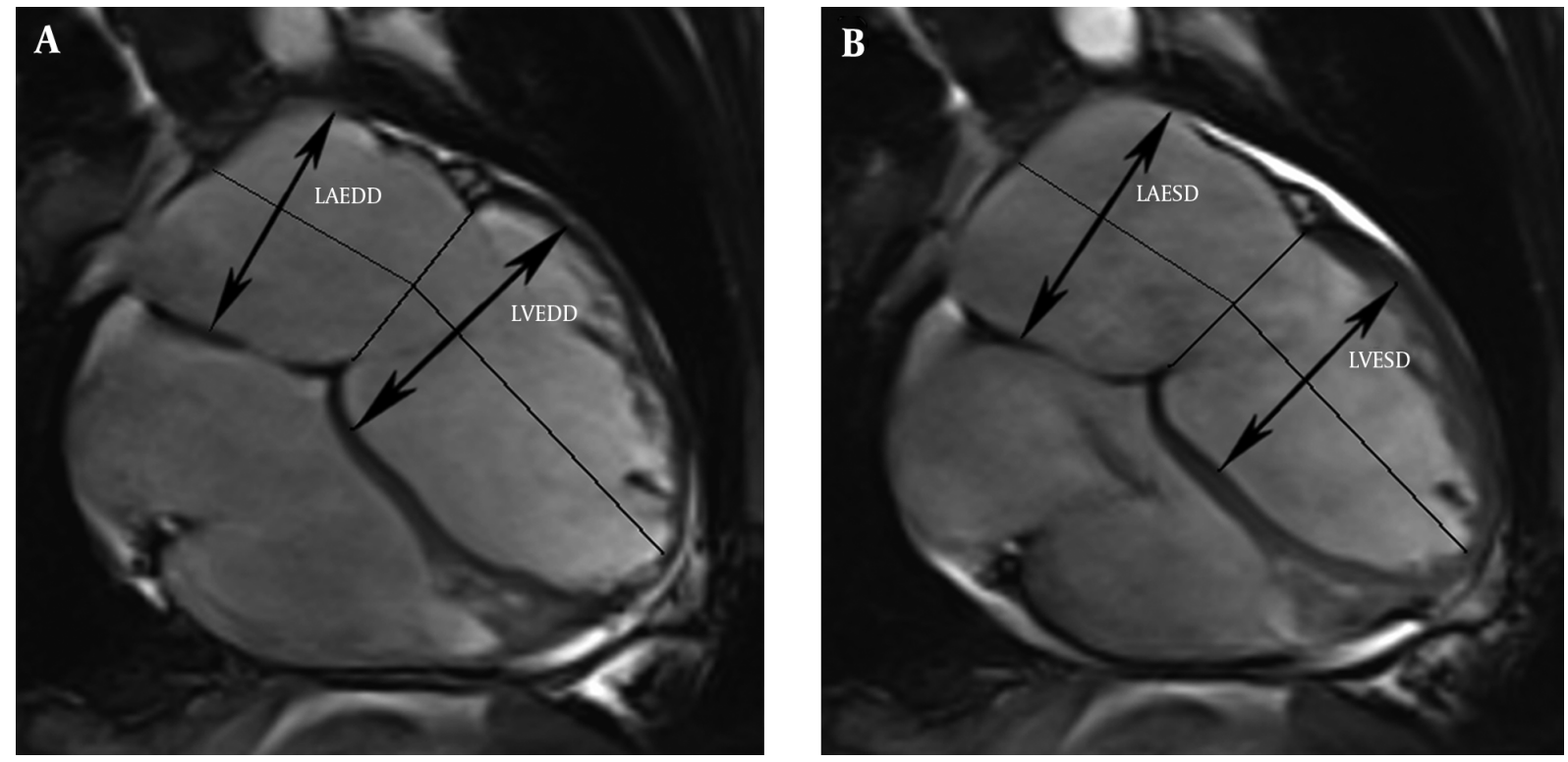

Figure 1. Measurement of the left atrium and left ventricular dimensions from four-chamber views in the end-diastole (A) and end-systole (B). (LAEDD, left atrial end-diastolic dimension; LVEDD, left ventricular end-diastolic dimension; LAESD, left atrial end-systolic dimension; LVESD, left ventricular end-systolic dimension).
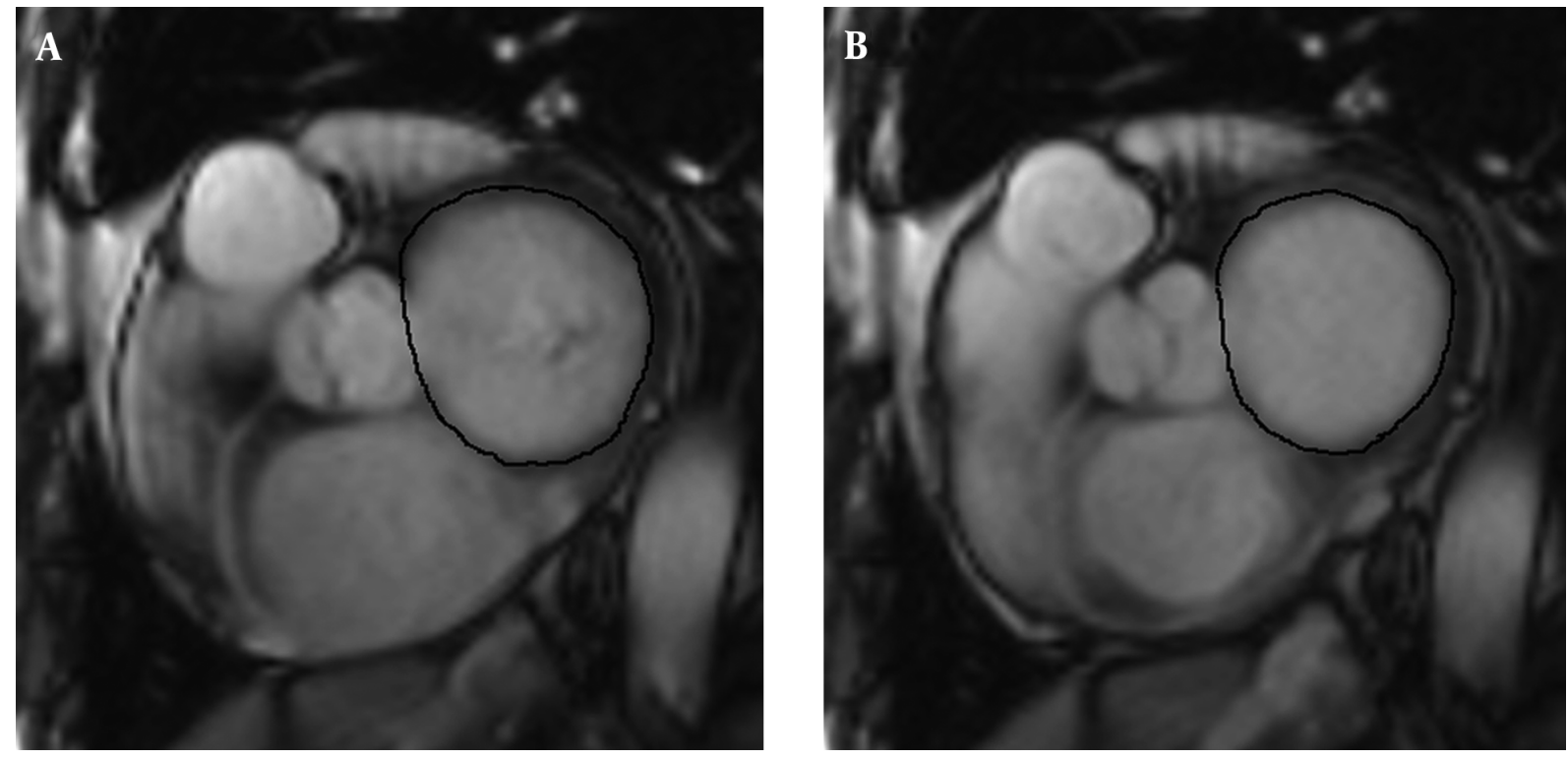

Figure 2. Planimetry of the $\mathrm{MAA}_{\max }(\mathrm{A})$ and $\mathrm{MAA}_{\min }(\mathrm{B})$. (black loop)

value (93.8\%) and sensitivity (94.4\%) were obtained when the indexed LVESD was greater than $3.4 \mathrm{~cm} / \mathrm{m}^{2}$. When a combination of the indexed LAESD, LVESD and MAA min $_{\text {was }}$ used, the specificity was increased to $91.3 \%$.

\subsection{Variability of MRI Measurement}

The interobserver and intraobserver variability showed an excellent agreement among MRI measurements as follows: the ICC of intraobserver variabilities for measurement of indexed LVEDV, LVESV, LAEDD, LAESD, and $\mathrm{MAA}_{\min }$ were 0.97, 0.95, 0.97, 0.95, and 0.92, respectively; 
Table 1. Baseline Characteristics of DCM Patients and Normal Individuals ${ }^{\mathrm{a}}$

\begin{tabular}{|c|c|c|c|}
\hline Parameter & DCM Patients $(n=41)$ & Healthy Subjects $(\mathrm{n}=26)$ & $\mathbf{P}$ \\
\hline Age, $y$ & $43 \pm 18$ & $39 \pm 14$ & 0.39 \\
\hline Men, No. (\%) & $29(70.7)$ & $14(53.9)$ & 0.21 \\
\hline BSA, $\mathbf{m}^{2}$ & $1.6 \pm 0.2$ & $1.6 \pm 0.1$ & 0.98 \\
\hline BMI, $\mathrm{kg} / \mathrm{m}^{2}$ & $21.9 \pm 4.6$ & $22.7 \pm 3.6$ & 0.62 \\
\hline Systolic pressure & $116.9 \pm 17.5$ & $122 \pm 5.9$ & 0.10 \\
\hline Diastolic pressure & $74.8 \pm 11.9$ & $79 \pm 4.6$ & 0.19 \\
\hline NYHA, I, II, III, IV & $5 / 8 / 16 / 12$ & - & - \\
\hline
\end{tabular}

Abbreviations: BSA, body surface area; BMI, body mass index; DCM, dilated cardiomyopathy; NYHA, New York heart association; y, year.

${ }^{\mathrm{a}}$ Values are expressed as mean $\pm \mathrm{SD}$ or No. $(\%)$

Table 2. Comparison of MRI Parameters Between DCM Patients and Healthy Subjects

\begin{tabular}{|c|c|c|c|}
\hline Parameters & Healthy Subjects & DCM Patients & P Value \\
\hline \multicolumn{4}{|l|}{ LV and LA Parameter } \\
\hline Indexed LVEDV, $\mathrm{mL} / \mathrm{m}^{2}$ & $71.5 \pm 11.0$ & $163.8 \pm 73.8$ & $<0.001$ \\
\hline Indexed LVESV, $\mathrm{mL} / \mathrm{m}^{2}$ & $27.6 \pm 5.7$ & $131.8 \pm 74.3$ & $<0.001$ \\
\hline $\operatorname{LVEF}(\%)$ & $61.3 \pm 6.6$ & $23.6 \pm 11.7$ & $<0.001$ \\
\hline Indexed LAEDV, mL/m² & $45.2 \pm 14.1$ & $84.6 \pm 37.3$ & 0.001 \\
\hline Indexed LA ESV, mL/m² & $19.7 \pm 5.7$ & $59.2 \pm 36.2$ & 0.001 \\
\hline Indexed LVEDD, $\mathrm{cm} / \mathrm{m}^{2}$ & $2.8 \pm 0.4$ & $4.2 \pm 0.8$ & $<0.001$ \\
\hline Indexed LVESD, $\mathrm{cm} / \mathrm{m}^{2}$ & $1.7 \pm 0.2$ & $3.7 \pm 1.9$ & $<0.001$ \\
\hline Indexed LAEDD, $\mathrm{cm} / \mathrm{m}^{2}$ & $2.4 \pm 0.4$ & $4.1 \pm 0.8$ & $<0.001$ \\
\hline Indexed LAESD, $\mathrm{cm} / \mathrm{m}^{2}$ & $1.7 \pm 0.3$ & $3.3 \pm 0.8$ & $<0.001$ \\
\hline \multicolumn{4}{|l|}{ Mitral valve parameter, $\mathrm{cm}^{2} / \mathrm{m}^{2}$} \\
\hline Indexed $\mathrm{MAA}_{\min }$ & $5.9 \pm 1.2$ & $9.9 \pm 4.0$ & 0.001 \\
\hline Indexed $\mathrm{MAA}_{\max }$ & $9.5 \pm 1.1$ & $12.9 \pm 3.8$ & 0.002 \\
\hline
\end{tabular}

Abbreviations: DCM, dilated cardiomyopathy; EDV, end-diastolic volume; ESV, end-systolic volume; EDD, end-diastolic diameter; ESD, end-systolic diameter; LV, left ventricle; LA, left atrium; LVEF, Left ventricular ejection fraction; MAA, mitral annulus area.

${ }^{\mathrm{a}}$ Values are expressed as mean $\pm \mathrm{SD}$

the ICC of interobserver variabilities for measurement of indexed LVEDV, LVESV, LAEDD, LAESD, and MAA $A_{\text {min }}$ were $0.95,0.96,0.98,0.91$, and 0.89 , respectively.

\section{Discussion}

In this study, we found that mitral regurgitation frequently coexists in DCM patients. With increasing severity of mitral regurgitation, the indexed LVESD, LAESD and $\mathrm{MAA}_{\min }$ tended to increase accordingly and might help to distinguish no/mild mitral regurgitation patients from moderate/severe mitral regurgitation patients.

DCM is a disease with multiple etiologies and morphological features. The chief characteristic of DCM is dilation of all chambers and impaired function of one or both ventricles, especially the left ventricle. Additionally, mild focal scarring of the mitral valve and dilation of the annulus are frequently observed $(1,16)$. In our study, echocardiographic examinations revealed that the proportion of DCM patients with mitral regurgitation was $73 \%$. The proportion of moderate/severe mitral regurgitation patients in our study was $44 \%$ (18/41), which was much higher than in Anwar's report $(20 \%, 4 / 20)$. (17). The reason for this difference could be different demographics of the patients.

As the most crucial systolic function parameter, EF is of great clinical importance during diagnosis, therapeutic assessment and prognostic prediction of cardiovascular disease. In our study, we found that EF in DCM pa- 
A

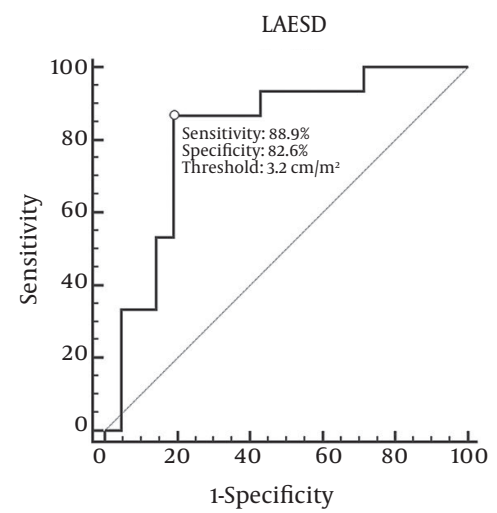

B

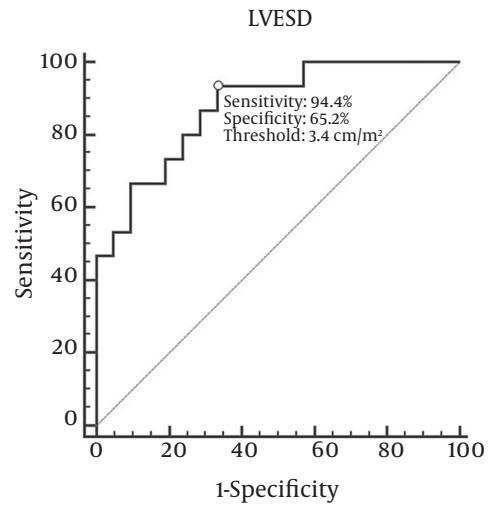

C

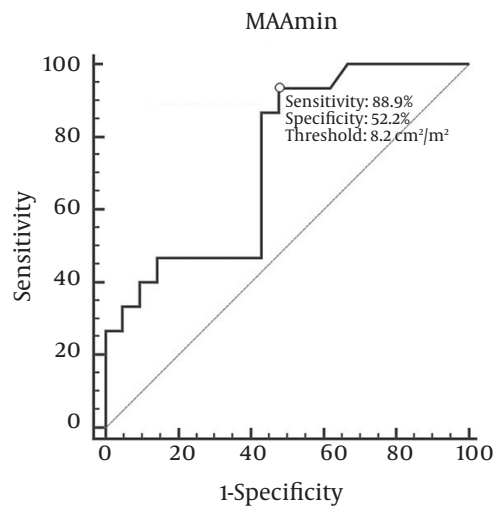

Figure 3. Receiver operating characteristic plot for predicting moderate/severe mitral regurgitation. A, Sensitivity and specificity of LA enlargement (LAESD> $3.2 \mathrm{~cm} / \mathrm{m}^{2}$ ) for diagnosis of moderate/severe mitral regurgitation were $88.9 \%$ and $82.6 \%$, respectively. B, Sensitivity and specificity of LV enlargement (LVESD> $3.4 \mathrm{~cm} / \mathrm{m}^{2}$ ) for diagnosis of moderate/severe mitral regurgitation were $94.4 \%$ and $65.2 \%$, respectively. C, Sensitivity and specificity of $\mathrm{MAA}_{\min }$ enlargement $\left(\mathrm{MAA}_{\mathrm{min}}>8.2 \mathrm{~cm}^{2} / \mathrm{m}^{2}\right)$ for diagnosis of moderate/severe mitral regurgitation were $77.8 \%$ and $91.3 \%$, respectively. (LAESD, left atrial end-systolic dimension; LVESD, left ventricular end-systolic dimension; MAA min, minimum mitral annulus area)

Table 3. MRI Parameters in DCM Patients with Different Severity of Mitral Regurgitation ${ }^{\mathrm{a}}$

\begin{tabular}{|c|c|c|c|c|}
\hline Parameters & No/Mild & Moderate/Severe & $\mathbf{P}$ & Spearman Correlation $\mathbf{r}(\mathbf{p})$ \\
\hline Patients (n) & 23 & 18 & & \\
\hline \multicolumn{5}{|l|}{ LV and LA parameter } \\
\hline Indexed LVEDV, $\mathrm{mL} / \mathrm{m}^{2}$ & $134.0 \pm 35.8$ & $205.5 \pm 92.6$ & 0.003 & $0.44(0.007)$ \\
\hline Indexed LVESV, $\mathrm{mL} / \mathrm{m}^{2}$ & $101.2 \pm 40.7$ & $174.6 \pm 89.8$ & 0.002 & $0.43(0.01)$ \\
\hline $\operatorname{LVEF}(\%)$ & $26.6 \pm 13.5$ & $19.3 \pm 7.5$ & 0.06 & $-0.38(0.022)$ \\
\hline Indexed LAEDV, $\mathrm{mL} / \mathrm{m}^{2}$ & $73.7 \pm 34.6$ & $100.0 \pm 36.8$ & 0.04 & $0.52(0.001)$ \\
\hline Indexed LAESV, $\mathrm{mL} / \mathrm{m}^{2}$ & $48.8 \pm 35.3$ & $73.7 \pm 33.2$ & 0.04 & $0.52(0.001)$ \\
\hline Indexed LV EDD, $\mathrm{cm} / \mathrm{m}^{2}$ & $3.8 \pm 0.6$ & $4.7 \pm 0.9$ & 0.003 & $0.44(0.006)$ \\
\hline Indexed LVESD, $\mathrm{cm} / \mathrm{m}^{2}$ & $3.2 \pm 0.7$ & $4.3 \pm 0.8$ & 0.001 & $0.58(0.000)$ \\
\hline Indexed LAEDD, $\mathrm{cm} / \mathrm{m}^{2}$ & $3.8 \pm 0.6$ & $4.7 \pm 0.9$ & 0.001 & $0.45(0.006)$ \\
\hline Indexed LAESD, cm $/ \mathrm{m}^{2}$ & $3.0 \pm 0.8$ & $3.8 \pm 0.6$ & 0.001 & $0.61(0.000)$ \\
\hline \multicolumn{5}{|l|}{ Mitral Valve Parameter, $\mathrm{cm}^{2} / \mathrm{m}^{2}$} \\
\hline Indexed $M A A_{\min }$ & $8.3 \pm 3.0$ & $12.1 \pm 4.3$ & 0.003 & $0.56(0.000)$ \\
\hline Indexed $\mathrm{MAA}_{\max }$ & $11.6 \pm 2.9$ & $14.8 \pm 4.1$ & 0.009 & $0.44(0.008)$ \\
\hline
\end{tabular}

Abbreviations: DCM, dilated cardiomyopathy; ESD, end-systolic dimension; EDV, end-diastolic volume; EF, ejection fraction; ESV, volumes in end- systole volume; EDD, end-diastolic dimension; LV, left ventricle; LA, left atrium; MAA, mitral annulus areas.

${ }^{\mathrm{a}}$ Values are expressed as mean $\pm \mathrm{SD}$.

tients with moderate/severe mitral regurgitation significantly decreased compared to that of no/mild mitral regurgitation patients, which was consistent with previous studies $(18,19)$. The impaired LV systolic function always occurs in concomitance with mitral regurgitation in DCM. The decreased EF in DCM frequently accompanies ventricular enlargement, resulting in deformation of the mitral complex, especially the mitral annulus. LV volume overload with increased preload secondary to mitral regurgitation hemodynamically affect EF. DCM patients with moderate/severe mitral regurgitation showed a larger LV volume and dimension, increasing the LV preload more prominently than no/mild patients, which in turn significantly decreased EF. 
Table 4. Prediction of Moderate/Severe Mitral Regurgitation in DCM Patients with Morphologic Parameters ${ }^{\mathrm{a}}$

\begin{tabular}{|c|c|c|c|c|c|}
\hline Morphologic Parameter & Sensitivity (\%) & Specificity (\%) & Accuracy (\%) & Positive Predictive Value (\%) & Negative Predictive Value (\%) \\
\hline LAESD $>3.2, \mathrm{~cm} / \mathrm{m}^{2}(1)$ & $88.9(16 / 18)$ & $82.6(19 / 23)$ & $85.4(35 / 41)$ & $80(16 / 20)$ & $90.5(19 / 21)$ \\
\hline LVESD $>3.4, \mathrm{~cm} / \mathrm{m}^{2}(2)$ & $94.4(17 / 18)$ & $65.2(15 / 23)$ & $78.0(32 / 41)$ & $68.0(17 / 25)$ & $93.8(15 / 16)$ \\
\hline MAAmin $>8.2, \mathrm{~cm}^{2} / \mathrm{m}^{2}(3)$ & $88.9(16 / 18)$ & $52.2(12 / 23)$ & $68.3(28 / 41)$ & $59.3(16 / 27)$ & $85.7(12 / 14)$ \\
\hline$(1)+(2)$ & $88.9(16 / 18)$ & $87.0(20 / 23)$ & $87.8(36 / 41)$ & $84.2(16 / 19)$ & $91.0(20 / 22)$ \\
\hline$(1)+(2)+(3)$ & $77.8(14 / 18)$ & $91.3(21 / 23)$ & $73.2(35 / 41)$ & $87.5(14 / 16)$ & $84(21 / 25)$ \\
\hline
\end{tabular}

Abbreviations: DCM, dilated cardiomyopathy; LAESD, left arterial end-systolic dimension; LVESD, left ventricular end-systolic dimension; MAA, mitral annulus areas.

${ }^{\mathrm{a}}$ Values are expressed as percentage (number of patients).

The normal closure of the mitral valve requires good coordination of the LA, LV, mitral annulus, mitral leaflets and the subvalvular apparatus (20). In DCM patients, chamber dilatation and alteration in the geometric relationship of the mitral valve apparatus may lead to mitral regurgitation, particularly MAA dilatation. According to previous studies $(2,3,21)$, the presence of mitral regurgitation is associated with a poor outcome, and a moderate/severe degree of mitral regurgitation remains an independent predictor for sudden cardiac death, heart failure, and mortality in DCM patients. Surgical correction for severe mitral regurgitation can improve patients' symptoms and quality of life, reduce hospital mortality, and reveal reverse LV remodeling $(14,22)$. Thus, morphological and function assessment of LA, LV, and MAA plays a key role in the therapeutic evaluation of DCM patients with significant mitral regurgitation. Precise measurement of MAA can help clinicians to choose appropriate prosthetic mitral valve rings and percutaneous annuloplasty devices $(23,24)$.

Although echocardiography is available and widely used for the diagnosis of DCM and LV function assessment, the low spatial resolution and operator-dependent characteristics hamper the accuracy quantification of parameters, especially volumetric parameters (25). MRI has been proven of clinical value for assessment of cardiac function and structure $(7,8,26)$. The unique challenges posed by cardiac imaging such as physiological motion and position of the heart can decrease image quality, especially SNR in low filed MRI $(8,27)$. Three tesla MRI overcomes the limitation of 1.5 T MRI and extends the capabilities of cardiac MRI. As the magnetic field strength increases, the bulk magnetization increases as well, resulting in a linear increasing of SNR (8). The improvement in SNR supports excellent delineation of the blood-myocardium interface and hence facilitates precise quantification of cardiac function. In addition, 3.0 T MRI allows reproducible and accurate measurement of detailed anatomy of the left heart such as the left ventricular, LA, and mitral valve $(15,17)$. These advancements of MRI may be beneficial for assessing functional and morphological features of the left heart and predicting the severity of coexistence of mitral regurgitation in DCM patients.

Previous studies have debated whether mitral regurgitation is caused by dysfunction of the ventricle, mitral valve or atrium $(4-6,28)$. In our study, we have found that all morphological and functional parameters of the LV, LA, and mitral valve in DCM patients with moderate/severe mitral regurgitation were greater than that of no/mild mitral regurgitation patients, and the indexed LVESD, LAESD and $\mathrm{MAA}_{\text {min }}$ tended to increase with increasing grades of mitral regurgitation. Furthermore, there was a significant correlation between indexed LVESD and LAEDD in patients with DCM. This suggested that DCM patients with a decreased LVEF had an increased risk proportional to increase in LA size due to the increasing after load. Similarly, previous reports have found that the main determinant of LA volume is ventricular diastolic function. Diastolic dysfunction should be regarded as an important contributing factor to the development of mitral regurgitation in DCM patients, likely by causing enlargement of LA and mitral annulus (4). Therefore, our results further confirmed that a combination of dilation and impaired function of the left heart with a larger MAA contribute to the occurrence and development of mitral regurgitation in DCM patients.

Recognition of the presence and severity of mitral regurgitation, especially a moderate/severe degree of mitral regurgitation, can be of great value for risk stratification, treatment decisions and prognosis prediction (2, 3,21 ). Our studies have revealed that the indexed LVESD, LAESD, and $\mathrm{MAA}_{\text {min }}$ are positively correlated with increasing grades of mitral regurgitation grading, and in particular, the indexed LVESD is sufficiently sensitive to detect moderate/severe mitral regurgitation. A combination of indexed LAESD and LVESD produced relatively higher sensitivity, specificity, positive predictive value, and negative predictive value. Further combination of indexed $\mathrm{MAA}_{\min }$ with LAESD and LVESD produced the highest specificity (91.3\%) for predicting mitral regurgitation severity. More- 
over, inter-and intraobserver variability analysis indicated that 3.0 T MRI appears to be an accurate and reliable modality for measuring these morphological parameters.

We acknowledge the following limitations of our study. A unicenter design allows for a consistent scanning protocol and excellent reproducibility in MRI but may also cause selection bias. The diagnostic value of the LV, LA and mitral valve in DCM patients with mitral regurgitation needs to be proven in larger cohort studies. Furthermore, the prognostic role of dysfunction and morphological measurements on cardiac MRI for survival in DCM needs further clinical investigation.

In conclusion, dysfunction and dilation of LV, LA, and mitral annulus in DCM patients with mitral regurgitation can be reliably and accurately determined by MRI. The in-

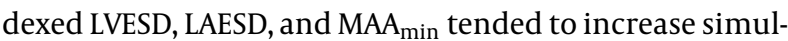
taneously with increasing grades of mitral regurgitation, which can assist in the prediction of mitral regurgitation severity with high sensitivity and specificity.

\section{Acknowledgments}

None.

\section{Footnotes}

Authors' Contributions: Zhi-gang Yang and Ying-kun Guo contributed equally to this work and should be considered co-corresponding authors. Study concept and design, Zhi-gang Yang, and Ling-yi Wen; acquisition of data, Ling-yi Wen, Hua-yan Xu, and Jia-yu Sun; analysis and interpretation of data, Ling-yi Wen, and Ying-kun Guo; drafting of the manuscript, Ling-yi Wen; critical revision of the manuscript for important intellectual content, Ying-kun Guo, and Zhi-gang Yang; statistical analysis, Ling-yi Wen, and Hua-yan Xu; study supervision, Zhi-gang Yang, and Ying-Kun Guo

Financial Disclosure: The authors have no financial disclosures.

Funding/Support: This work was funded by the national natural science foundation of China (81271625, 81471721, 81471722).

\section{References}

1. Jefferies JL, Towbin JA. Dilated cardiomyopathy. Lancet. 2010;375(9716):752-62. doi: 10.1016/S0140-6736(09)62023-7. [PubMed: 20189027].

2. Bursi F, Barbieri A, Grigioni F, Reggianini L, Zanasi V, Leuzzi C, et al. Prognostic implications of functional mitral regurgitation according to the severity of the underlying chronic heart failure: a longterm outcome study. EurJ Heart Fail. 2010;12(4):382-8. doi: 10.1093/eurjhf/hfq014. [PubMed: 20197266].
3. Agricola E, Stella S, Figini F, Piraino D, Oppizzi M, D’Amato R, et al. Non-ischemic dilated cardiopathy: prognostic value of functional mitral regurgitation. Int J Cardiol. 2011;146(3):426-8. doi 10.1016/j.ijcard.2010.10.096. [PubMed: 21094544].

4. Park S, Park S, Casaclang-Verzosa G, Ommen S, Pellikka P, Miller F, et al. Diastolic dysfunction and left atrial enlargement as contributing factors to functional mitral regurgitation in dilated cardiomyopathy: data from the Acorn trial. Am heart J. 2009;157(4):762.

5. Fernandez-Golfin C, De Agustin A, Manzano MC, Bustos A, Sanchez T, Perez de Isla L, et al. Cardiac magnetic resonance determinants of functional mitral regurgitation in ischemic and non ischemic left ventricular dysfunction. Int J Cardiovasc Imaging. 2011;27(4):539-46. doi: 10.1007/s10554-010-9696-y. [PubMed: 20824507].

6. Lesniak-Sobelga A, Wicher-Muniak E, Kostkiewicz M, Olszowska M, Musialek P, Klimeczek P, et al. Relationship between mitral leaflets angles, left ventricular geometry and mitral deformation indices in patients with ischemic mitral regurgitation: imaging by echocardiography and cardiac magnetic resonance. Int J Cardiovasc Imaging. 2012;28(1):59-67. doi: 10.1007/s10554-010-9783-0. [PubMed: 21213048].

7. Pennell DJ. Cardiovascular magnetic resonance. Circulation. 2010;121(5):692-705. doi: 10.1161/CIRCULATIONAHA.108.811547. [PubMed: 20142462].

8. Oshinski JN, Delfino JG, Sharma P, Gharib AM, Pettigrew RI. Cardiovascular magnetic resonance at 3.0 T: current state of the art. J Cardiovasc Magn Reson. 2010;12:55. doi: 10.1186/1532-429X-12-55. [PubMed: 20929538].

9. Shimada YJ, Shiota T. A meta-analysis and investigation for the source of bias of left ventricular volumes and function by three-dimensional echocardiography in comparison with magnetic resonance imaging. Am J Cardiol. 2011;107(1):126-38. doi: 10.1016/j.amjcard.2010.08.058. [PubMed: 21146700].

10. Burianova L, Riedlbauchova L, Lefflerova K, Marek T, Lupinek P, Kautznerova D, et al. Assessment of left ventricular function in nondilated and dilated hearts: comparison of contrast-enhanced 2dimensional echocardiography with multi-detector row CT angiog raphy. Acta Cardiol. 2009;64(6):787-94. doi: 10.2143/AC.64.6.2044744 [PubMed: 20128156].

11. Lang RM, Bierig M, Devereux RB, Flachskampf FA, Foster E, Pellikka PA, et al. Recommendations for chamber quantification. Eur JEchocar diogr. 2006;7(2):79-108. doi: 10.1016/j.euje.2005.12.014. [PubMed: 16458610].

12. Bogaert J, Dymarkowski S, Taylor AM, Muthurangu V. Clinical cardiac MRI. 2 ed. Springer-Verlag Berlin Heidelberg; 2012.

13. Bonow RO, Carabello BA, Chatterjee K, de Leon AJ, Faxon DP, Freed MD, et al. 2008 focused update incorporated into the ACC/AHA 2006 guidelines for the management of patients with valvular heart disease: a report of the American College of Cardiology/American Heart Association Task Force on Practice Guidelines (Writing Committee to revise the 1998 guidelines for the management of patients with valvular heart disease). Endorsed by the Society of Cardiovascular Anesthesiologists, Society for Cardiovascular Angiography and Interventions, and Society of Thoracic Surgeons. J Am Coll Cardiol. 2008;52(13):1-142. doi: 10.1016/j.jacc.2008.05.007. [PubMed: 18848134].

14. De Bonis M, Taramasso M, Verzini A, Ferrara D, Lapenna E, Calabrese $\mathrm{MC}$, et al. Long-term results of mitral repair for functional mitral regurgitation in idiopathic dilated cardiomyopathy. Eur J Cardiothorac Surg. 2012;42(4):640-6. doi:10.1093/ejcts/ezs078. [PubMed: 22447810]

15. Sievers B, Kirchberg S, Addo M, Bakan A, Brandts B, Trappe HJ. Assessment of left atrial volumes in sinus rhythm and atrial fibrillation using the biplane area-length method and cardiovascular magnetic resonance imaging with TrueFISP. J Cardiovasc Magn Reson. 2004;6(4):855-63. [PubMed: 15646889].

16. Dec GW, Fuster V. Idiopathic dilated cardiomyopathy. N Engl J Med 1994;331(23):1564-75. doi: 10.1056/NEJM199412083312307. [PubMed: 7969328]. 
17. Anwar AM, Soliman OI, Nemes A, Germans T, Krenning BJ, Geleijnse ML, et al. Assessment of mitral annulus size and function by real-time 3-dimensional echocardiography in cardiomyopathy: comparison with magnetic resonance imaging. J Am Soc Echocardiogr. 2007;20(8):941-8. doi: 10.1016/j.echo.2007.01.026. [PubMed: 17555937].

18. Trichon BH, Felker GM, Shaw LK, Cabell CH, O'Connor CM. Relation of frequency and severity of mitral regurgitation to survival among patients with left ventricular systolic dysfunction and heart failure. Am J Cardiol. 2003;91(5):538-43. [PubMed: 12615256].

19. Stolfo D, Merlo M, Pinamonti B, Poli S, Gigli M, Barbati G, et al. Early improvement of functional mitral regurgitation in patients with idiopathic dilated cardiomyopathy. Am JCardiol. 2015;115(8):1137-43. doi: 10.1016/j.amjcard.2015.01.549. [PubMed: 25721482].

20. Perloff JK, Roberts WC. The mitral apparatus. Functional anatomy of mitral regurgitation. Circulation. 1972;46(2):227-39. [PubMed: 5046018].

21. Patange A, Thomas R, Ross RD. Severity of mitral regurgitation predicts risk of death or cardiac transplantation in children with idiopathic dilated cardiomyopathy. Pediatr Cardiol. 2014;35(2):232-8. doi: 10.1007/s00246-013-0764-7. [PubMed: 23917522].

22. Acker MA, Jessup M, Bolling SF, Oh J, Starling RC, Mann DL, et al. Mitral valve repair in heart failure: five-year follow-up from the mitral valve replacement stratum of the Acorn randomized trial. J Thorac Cardiovasc Surg. 2011;142(3):569-74. doi:10.1016/j.jtcvs.2010.10.051. [PubMed:
21269649].

23. Bothe W, Miller DC, Doenst T. Sizing for mitral annuloplasty: where does science stop and voodoo begin?. Ann Thorac Surg. 2013;95(4):1475-83. doi: 10.1016/j.athoracsur.2012.10.023. [PubMed: 23481703].

24. Sack S, Kahlert P, Bilodeau L, Pièrard L, Lancellotti P, Legrand V, et al. Percutaneous transvenous mitral annuloplasty initial human experience with a novel coronary sinus implant device. Circulation: Cardiovas Intervent. 2009;2(4):277-84.

25. Guo YK, Yang ZG, Ning G, Rao L, Dong L, Pen Y, et al. Sixty-four-slice multidetector computed tomography for preoperative evaluation of left ventricular function and mass in patients with mitral regurgitation: comparison with magnetic resonance imaging and echocardiography. Eur Radiol. 2009;19(9):2107-16. doi: 10.1007/s00330-009-13928. [PubMed: 19350247].

26. Bogaert J. Heart muscle diseases. Springer; 2014. pp. 275-98.

27. Lee VS, Hecht EM, Taouli B, Chen Q, Prince K, Oesingmann N. Body and cardiovascular MR imaging at 3.0 T. Radiology. 2007;244(3):692-705. doi: 10.1148/radiol.2443060582. [PubMed: 17709825].

28. Yiu SF, Enriquez-Sarano M, Tribouilloy C, Seward JB, Tajik AJ. Determinants of the degree of functional mitral regurgitation in patients with systolic left ventricular dysfunction: A quantitative clinical study. Circulation. 2000;102(12):1400-6. [PubMed: 10993859]. 